without insulin but who now enjoy decades of active life.

But what has all this got to do with medical ethics? Well, if Inglis's accusations are justified we are a pretty amoral lot. He suspects that we deliberately neglect our patients' psycho-social problems because of an erroneous mechanistic theory of specific aetiology of disease. He claims we refuse to allow them to be more responsible for their own treatment, and also that we impose a sense of blame on them when we diagnose a psychogenic basis for any illness. Furthermore, we collaborate with the drug industry in the promotion and misuse of endless new varieties of potentially harmful drugs, trying them out as if our patients were guinea pigs. Our ethical committees he dismisses as ineffective: our controlled trials are pseudo-scientific and unreliable, (largely because of inconsistencies in trials of psychotropic drugs and ECT). Our increasing specialisation encourages competition for ever more elaborate equipment for diagnosis and treatment when we should be spending more time in understanding our patients' 'real' problems as whole people and not just as disordered machines. All this evil inevitably rubs off on medical students so that our sins are self-propagating.

Readers of Ian Kennedy's Reith lectures and the controversy that they engendered in volume 7 of this journal (pp I73-2I I) will recognise that even if Inglis's attack is exaggerated there is some truth underlying it. Many doctors who read this book may agree that they need to improve their understanding of their patients, but they will be irritated and annoyed, as I was, by the lack of any mention of all that is good and beneficial in modern scientific medical and surgical practice. They will also be disappointed if they reach the final chapter, 'The Way Ahead', for they will find no clear signposts to show how the psycho-social problems which cause disease may be alleviated. Nor will they agree to full recognition of practitioners of 'Fringe medicine', nor to lay control of research funds to channel them away from the useless projects of, for instance, cancer experts. The final plea that we should 'achìve a better understanding of ourselves through a liberation of instincts long dormant, and where necessary a change in our lifestyles' is not one on which action can readily be taken or success achieved.

Despite its bias, omissions and inaccuracies I found this book did present a challenge with an ethical component to which the rising generation of doctors may pay more attention than their teachers are likely to pay.

CHARLES FLETCHER 20 Drayton Gardens London

\section{Treating The Troublesome}

\section{Council for Science and Society Report, London \\ $£_{2.50}$}

The Council for Science and Society was formed in 1973 with the object of 'promoting the study of, and research into, the social effects of science and technology, and of disseminating the results thereof to the public'. This report, produced by a working party convened by David Sullivan QC, describes techniques of treatment currently used to modify behaviour or personality and examines the ethical problems of giving such treatments, with or without consent.

The ten members of the working party are to be congratulated on producing a literate and lucid report which reflects the application of much liberal and humane thought to a most difficult problem: when should treatment be compulsory? They prepare the ground for approaching this question by starting with an informative and balanced section on the physical and psychological techniques of treatment that are available.

A section on places of confinement rightly draws attention to the extreme pressures on the four special hospitals and on the prison service, pressures which inevitably push the management of mentally ill offenders in an authoritarian and restrictive direction, where there is a greater risk of professional ethics being stretched by the exigencies of administering grossly under-staffed institutions. The pressures might be reduced if the Regional Health Authorities could be persuaded to provide the 'medium security' units they have been instructed and funded to provide. This working party also makes the recommendation ( $\mathrm{p} 48$ ) 'that a small number of well-equipped prison hospitals should be designated as mental hospitals, so that prisoners could be detained there under a Mental Health Act certificate'.

The money spent on the prison service and on the mentally ill of all categories needs to be greatly increased if any appreciable impact is going to be made on the problem of recidivism and anti-social mental disorder. Society behaves like an ostrich, trying to shut away and ignore the troublesome patient, a course of action which is not only inhumane but, in the long run, more expensive than tackling the problem.

Ideally, professional ethical codes provide a safeguard against the abuse of treatment without consent. But not all professions have codes that are as rigorously enforced as that of the medical profession. Psychologists, for example, are expected to observe the code of practice of the British Psychological Society, but even if transgression leads to expulsion from the society a psychologist could still continue to practise. A similar situation exists for social workers and psychotherapists, although the statutory registration of psychotherapists has been actively discussed for some years and may well soon become a reality. The report also makes the valid point ( $\mathrm{p} \mathrm{26}$ ) 'that in Britain ethical education, which of course is difficult in that it is not a matter of didactive (sic) tuition but the fostering of an approach, is the exception rather than the rule in medical schools and postgraduate courses'.

The Mental Health Act of 1959 contains safeguards, and the Butler Committee of 1975 recommended further safeguards, some endorsed in the Mental Health (Amendment) Bill I98I, but not, at the time of writing, yet enacted. This working party makes a number of additional proposals, two being important in my view. Firstly, it proposes that as well as obtaining a professional second opinion whenever there is any doubt about the correct diagnosis or appropriate treatment, a lay second opinion should be obtained whenever ( $\mathrm{p} 44$ ) 'there is an important defect in the patient's understanding, or in his competence or freedom to give consent. What is needed in such cases is an opinion from someone who can represent the patient's interests, but who is not subject to his disabilities'. How closely this ideal could be approached remains to be seen. Secondly, it proposes a voluntary rather than compulsory system of maintaining safeguards, arguing that a compulsory system would be cumbersome and bureaucratic and would entail ( $p$ 45) 'the possibility of an effective veto over a clinician's freedom to treat his patient as he thinks best'.

It is a pity that the working party does not give some clinical examples to make 
its arguments more alive. It is also a pity that so little attention is given to children in the report: a pity because, in the realm of mental health, prevention or earlier intervention is so much better than later intervention. The statement on p I that 'far too little is yet known about the causes of [troublesome] behaviour' is misleading. Of course we must modestly acknowledge our ignorance, but we do know enough to know that there are a number of measures that society could take to improve the care of babies and young children, and to reduce further the number of unwanted children, thereby reducing the incidence of serious personality disorder and mental illness later in life, and preventing at least some of the ethical headaches that consequently arise. But that is something for another working party.

IAIN DRESSER

Department of Child Psychiatry Paddington Green Children's Hospital

London

\section{Death and Dying, a Quality of Life}

\author{
Eds Patricia F Pegg and Erno Metze \\ Pitman, London \\ £I 5
}

This book is made up from the papers presented at the International Congress on Death and Dying held in London in 1980. The proceedings of such gatherings are often disjointed and indigestible, but here is the exception which proves the rule. Having said that, it is easy to take issue with the order in which the papers are presented. One can sympathise with the editor's dilemma: is it best to proceed from the general to the particular and risk the reader losing interest in what appears to be the main thrust of the book or to start with the particular and risk not seeing the wood for the trees?

As the structure stands the first half of the book deals almost solely with bereavement and the reader began to wonder what the sub-title meant. It is not that the earlier papers are not interesting, but somehow they engendered a feeling of impatience because they seemed to be setting the scene at a pace reminiscent of the opening of a novel by Sir Walter Scott. No doubt on second reading the early chapters would come into their own; they are just in a different key from the middle movement of papers which are narrower in scope but have a freshness and immediacy which will quickly engage the attention of professionals working in the field. The final movement returns to bereavement but in more specific contexts which are of great interest and value.

Not that the early papers are unimportant or uninteresting. The primitive societies' rituals to placate the ghosts of the dead are seen to be equivalent to the painful disengagement of the bereaved member of a Western culture from the emotional bonds with the deceased. This not only illuminates what is happening for an interested professional, but also opens the way to understanding how communities respond to bereavement, which is described in the closing chapters.

Inevitably there is overlap, but this is not irritating. Manocchio's chapter covers most of the field, but the fact that there is a section on the stillborn child only serves to emphasise the importance of this problem (dealt with in two other papers) which, until recently, has been almost totally neglected by the professionals concerned. As in many other areas, only the personal experience of a professional of having a stillborn child may stimulate an enquiry into why stillbirths have been swept under the carpet. The same might also be said of cot-deaths. This section is particularly valuable for professionals who may only come across such disasters occasionally, and who therefore may feel at sea with the emotional problems of the parents. Sudden death in adults is much commoner and some experience may be available, but the chapters on sudden death and disasters are valuable for their analysis of the needs of the bereaved.

The chapters on coping with the fears of recurrence and death, together with Metze's chapter on the coronary patient living with the possibility of sudden death, are very exciting. They attempt to break new ground while at the same time remaining extremely practical. The concept of the need for the patient who has developed cancer recurrence to put his mind inte 'neutral' is fascinating and opens up an alternative to the paths of denial or defeat. The chapter is strengthened by the case histories.

These chapters are set off by the chapter by Schneider which seeks to conceptualise the process of bereavement and provides interesting and useful maps of the country through which the journey has to be undertaken.
This brief survey must inevitably seem to be invidious to those authors whose papers have not been touched on - notably absent are those from Antonius-Ijsselmonde - but this is only because the hospice concept is perhaps more firmly established in the UK and therefore appears less striking, although the Dutch workers are breaking new ground.

Altogether it is remarkable that a collection of authors working in different countries and on different lines can produce a satisfying feeling of synthesis in the mind of the reader. Perhaps it is a pity that the title and sub-title were not reversed!

\section{MICHAEL COURTENAY \\ Bridge Lane Health Centre, London SW11}

\section{Bereavement Visiting}

\section{Edited by G Dyne}

King's Fund Publishing Office, London

$$
£_{2.85}
$$

This attractively produced small book describes the bereavement visiting service at St Christopher's Hospice, London. It is not a textbook about grief, though the interested reader is given a number of suggestions about further reading. The service started in $197 \mathrm{I}$, initially in a small way. Visitors come from varied backgrounds but all have worked at the hospice in some capacity, either as a volunteer or on the staff. Volunteers make good visitors but, as with staff members, there is need for support. This is given mainly by a monthly review meeting. Access to a social worker and psychiatrist is also available should this be necessary.

Advice is given about arranging a visit and the reader is given an indication of what might happen during one. Three case studies add much to the value of the book. The final section describes the Pilgrim Club, an informal weekly meeting for the bereaved. All in all this is a helpful book for those working in similar settings. The need to care for the bereaved will always be with us.

ROBERT G TWYCROSS

Sir Michael Sobell House The Churchill Hospital Headington 